\title{
Increasing Species Diversity in Neuroscience Research: How and Why?
}

\author{
Georg F. Striedter ${ }^{\mathrm{a}}$ Todd M. Preuss ${ }^{\mathrm{b}}$ \\ a Department of Neurobiology and Behavior, University of California Irvine, Irvine, CA, USA; \\ ${ }^{b}$ Division of Neuropharmacology and Neurologic Diseases, Yerkes National Primate Research Center, \\ Emory University, Atlanta, GA, USA
}

This is an interesting time in comparative neurobiology. In some respects, pessimism is warranted. It is unfortunate, for example, that support for non-human primate research has diminished substantially in the USA, while studies on a single "model" species less closely related to humans - the laboratory mouse - has come to dominate neuroscience. Moreover, the heavy emphasis on mice, endorsed by funding agencies that broadly accept the assumption that results in mice will translate to treatments for human disorders, leaves little room for truly comparative studies conducted in a phylogenetic framework - including the search for alternative models that might prove useful for specific purposes. On a more optimistic note, Japan is investing heavily in research on marmoset monkeys, while China is boosting research on macaques. Moreover, comparative genomic studies are flourishing across the globe, and rapid technical advances increasingly enable researchers to manipulate the genomes of many different species, greatly facilitating both evo-devo and neuroethological research.

Against this backdrop, we organized the 30th Karger Workshop and this Special Issue to examine the how and why of species choice in neuroscience research. To that end, we invited a range of scholars who could address the history, theory, and practice of model choice in neuroscience. Specifically, we selected Cheryl Logan and Jessica Bolker, as well as the two of us, to discuss the history and theory of how researchers choose their study species. We

\section{KARGER}

(c) 2019 S. Karger AG, Basel

E-Mail karger@karger.com

www.karger.com/bbe also invited Barbara Finlay, who emphasized the importance of studying inter- and intraspecific variation, which yields useful principles of variation and has revealed, for example, that laboratory mice are rather deviant in multiple respects. Two additional participants were Melina Hale and Scott Juntti, who focused on the opportunities generated by technical advances in genome manipulation, and on the application of those cutting-edge techniques in a diverse array of species. Finally, Shreesh Mysore and Pasko Rakic presented new data obtained in two different "model" species, namely barn owls and laboratory mice, and then related their findings to data from other species.

Across this diverse set of perspectives, several themes emerged. One is that the phylogenetic scale and other fallacies continue to mislead research. Even the more reasonable notion that the degree of similarity between two species falls off with phylogenetic distance is only true on average: many exceptions to the rule exist. For example, marmosets are more similar to humans than macaques in terms of their breeding behavior, even though they are more distant relatives. This finding is likely to surprise most neuroscientists because they tend not to appreciate how common convergent evolution is. Such misconceptions about how evolution works should be addressed by giving neuroscientists more robust training in evolutionary neurobiology and asking them to think seriously about evolution in their research. Another recurring

Prof. Georg F. Striedter

Department of Neurobiology and Behavior

University of California Irvine, $2205 \mathrm{McGaugh}$ Hill

Irvine, CA 92697-4550 (USA)

E-Mail georg.striedter@gmail.com 
workshop theme was that comparative neurobiologists should more clearly articulate the value of studying a wide variety of species, including both invertebrates and vertebrates. Simply promoting the use of yet another model species merely reinforces the misguided notion that all the important features of biology are broadly conserved. Of course, some species may be more convenient for the study of some widely conserved traits, but variation across species can also yield useful information. Comparative research can, for example, reveal which features are restricted to a specific group of animals and which have evolved convergently in more than one. Moreover, it can reveal cross-species scaling rules that allow inferences about features that have not yet been studied in a species of interest (e.g., humans). Similarly useful is research on species that are resistant to particular diseases or pathogens, as they can provide important clues for therapy development.

A third general concern emerging from this workshop is that the current paradigm of how biological research is conducted imposes barriers to studying diverse species. As a given species assumes model status, networks of researchers and resources come to define a species-specific research community that tends, with growth, to become insular. To counteract this tendency toward scientific Balkanization, it will be important to continue the development of techniques that can be used in diverse species and to provide opportunities for test-driving those methods in strategically selected species. In addition, young scientists should be more thoroughly exposed to the awesome molecular, anatomical, physiological, and behavioral diversity of species. Given broad knowledge and opportunity, new investigators looking to distinguish them- selves will always want to apply their bag of tricks to new problems and, occasionally, new species. As their efforts contribute to an ever-growing tapestry of comparative data, it becomes easier to reconstruct phylogenetic histories, discover correlations between various traits and, more generally, link genotypes to phenotypes across development and evolutionary time. An exciting prospect, indeed!

In pursuit of this more inclusive, integrative comparative neurobiology, we call for more in-depth assessments of existing models, research on more diverse species, and the integration of comparative data into a comprehensive scheme that goes beyond a list of what is conserved. The last of these three objectives is challenging in part because conservation is generally (albeit dubiously) taken to be the "null hypothesis" and because it is more difficult to explain species differences than commonalities (which can simply be attributed to common descent, although one might well ask why they were so well conserved). However, biological evolution consists of descent with modification, and we should aim to understand both aspects of evolution. One benefit of taking variation into account is that it will improve translational efforts. An excellent example is the recent push by the NIH to recognize and scrutinize sex differences in both clinical and preclinical research. Many researchers initially balked at this effort, largely because it would require increases in sample size, but the benefit to women's health is now widely accepted. By analogy, we are optimistic that future generations of neuroscientists will be more mindful of species differences and integrate them into their conception of biology. We hope the papers in this Special Issue will make a contribution to this long-term goal. 